\title{
The role of CR3 (CD11b/CD18) and CR4 (CD11c/CD18) in complement- mediated phagocytosis and podosome formation by human phagocytes
} Szilvia Lukácsi ${ }^{\mathrm{a}}$, Zsuzsa Nagy-Baló ${ }^{\mathrm{a}}$, Anna Erdei ${ }^{\mathrm{a}, \mathrm{b}}$, Noémi Sándor ${ }^{\mathrm{b}, *}$, Zsuzsa Bajtay ${ }^{\mathrm{a},{ }^{*}}$

${ }^{a}$ Department of Immunology, Eötvös Loránd University, Pázmány P. s. 1/C, Budapest H1117, Hungary

${ }^{b}$ MTA-ELTE Immunology Research Group, Budapest, Eötvös Loránd University, Pázmány P. s. 1/C, Budapest H-1117, Hungary

*These authors contributed equally to this work.

\begin{abstract}
CR3 and CR4 belong to the family of $\beta_{2}$-integrins and play an important role in phagocytosis, cellular adherence and migration. CR3 and CR4 are generally expected to mediate similar functions due to their structural homology, overlapping ligand specificity and parallel expression on human phagocytes. Although the different signalling pathways of these receptors suggest distinct functions, possible differences are just being revealed.

Previously we proved that CR3 plays a key role in the uptake of iC3b-opsonized particles by human dendritic cells. Now, besides measuring the overall phagocytic capacity of cells including the assessment of surface bound as well as internalized particles, we extended our investigations and studied the digestion of the $\mathrm{iC} 3 \mathrm{~b}$ opsonized antigen by various human phagocytes. The participation of CR3 and CR4 was compared in the process of binding, internalization and digestion of iC3b opsonized Staphylococcus aureus by monocytes, monocyte derived macrophages (MDMs), monocyte derived dendritic cells (MDDCs), and neutrophils. Comparing the activity of the two $\beta_{2}$-integrin type complement receptors we found that CR3 plays a dominant role in the phagocytosis of iC $3 \mathrm{~b}$ opsonized S. aureus by all of these cell types.
\end{abstract}

Studying another important integrin-mediated function we demonstrated earlier that CR4 is dominant in the adhesion of monocytes, MDMs and MDDCs to fibrinogen. Here we studied the participation of CR3 and CR4 in podosome formation by human phagocytes, since these structures are known to play an essential role in cell migration. Our confocal microscopy analysis revealed that both $\mathrm{CD} 11 \mathrm{~b}$ and $\mathrm{CD} 11 \mathrm{c}$ concentrate in the podosome adhesion ring. 
In summary our data highlight differences in the function of human CR3 and CR4 in the process of uptake and digestion of complement opsonized antigen, while in the process of podosome formation, connected to cellular motility, both receptors equally take part.

\section{Keywords}

phagocytosis, podosomes, human phagocytes, $\beta_{2}$ integrins, CR3 (CD11b/CD18), CR4

(CD11c/CD18)

\section{Highlights}

- $\quad$ CR3 is the dominant receptor mediating the phagocytosis of $\mathrm{iC} 3 \mathrm{~b}$ opsonized S. aureus

- $\quad$ surface bound and digested bacteria should be distinguished

- MDDCs have a unique podosome pattern compared to monocytes, MDMs and neutrophils

- both CR3 and CR4 are located in the adhesion ring of podosomes

\section{Introduction}

Complement receptor CR3 (CD11b/CD18, also known as Mac-1; $\left.\alpha_{M} \beta_{2}\right)$ and CR4 $\left(\mathrm{CD} 11 \mathrm{c} / \mathrm{CD} 18\right.$, also known as p150, 95; $\alpha_{\mathrm{x}} \beta_{2}$ ) belong to the family of $\beta_{2}$ integrins containing a common $\beta$ chain and a different $\alpha$ subunit. The natural ligand of CR3 and CR4 is the inactivated fragment of C3 (iC3b),(Rosen and Law 1990; Ross and Vetvicka 1993) furthermore they also bind fibrinogen and ICAM-1 facilitating cellular adhesion (Lishko, 2001, Blood; Diamond, The Journal of Cell Biology,1993).

The best known functions of these receptors include cell adhesion and the clearance of pathogen microbes, tumour- and apoptotic cells through phagocytosis. Immune cells circulating in the bloodstream sense danger in the form of a chemoattractant gradient and move towards its source (Ley, 2007, Nature reviews) Tissue damage caused by injury or infection is followed by the deposition of fibrinogen, which plays an important role in regulating inflammatory responses. The engagement of fibrinogen via CR3 and CR4 enhances antimicrobial functions such as cell adhesion, cytokine and chemokine production (Flick, The Journal of clinical investigation, 2004; Thacker, Experimental and molecular pathology, 2008). In a previous study we demonstrated, that CR4 dominates adhesion to fibrinogen over CR3 in the case of human monocytes, MDMs and MDDCs (Sándor, plos one, 2016).

Integrins are known to play an important role in cell movement. Immune cells of the monocytic lineage use specific adhesive structures for migration, called podosomes. Intravascular crawling of monocytes, moving in the extracellular matrix after transmigration and dendritic cell movement to lymph nodes after antigen uptake are all achieved with the help of podosomes (Burns, Blood, 2001; Calle, European journal of cell biology, 2006), 
which are small, actin rich structures found on the contact surface of adherent cells. Their morphology distinguishes them from other adhesive structures since they contain an F-actin core which is surrounded by adhesion molecules (Calle, European journal of cell biology, 2006; Linder, Cellular and Molecular Life Sciences, 2015).

Activated cells leave the blood vessels with the help of integrin mediated connections and engage the invading pathogens. To capture and internalize targets, phagocytes utilize a range of surface receptors. Pattern recognition receptors bind the pathogens directly, while opsonized targets become recognizable by complement- and Fc-receptors, significantly increasing the efficiency of phagocytosis. (Dupuy \& Caron, 2008; Stuart \& Ezekowitz, 2005) Integrin mediated uptake can be also utilized for entry into host cells (BZs2004JI,Oliva 2008). Although professional phagocytes originate from a common myeloid progenitor cell, their role in immune defence differs greatly. (Stuart \& Ezekowitz, 2005). Monocytes, macrophages and neutrophil granulocytes show a higher capacity to internalize and kill bacteria than dendritic cells. The former cell types have evolved to effectively eliminate bacteria, whereas the main function of dendritic cells (DCs) is antigen presentation.(Nagl et al., 2002) Hence, both acidification and phagosomal degradation are lower in dendritic cells than in macrophages or neutrophils, enabling DCs to 'preserve' information derived from the pathogen. (Savina \& Amigorena, 2007)

Our group previously found that in the case of human MDDCs CR3 plays a dominant role in the phagocytosis of $\mathrm{iC} 3 \mathrm{~b}$ opsonized Staphylococcus aureus and Saccharomyces cerevisiae (Sándor, Immunobiology 2013). Due to the known differences between various phagocytes regarding the process of internalization and killing (Savina \& Amigorena, 2007), we set out to investigate human monocytes, MDMs and neutrophil granulocytes. In order to distinguish surface bound and digested bacteria, pHrodo Green conjugated bacteria were also used in addition to Alexa488 labelled $S$. aureus particles. The former one is a $\mathrm{pH}$ sensitive dye, which becomes highly fluorescent in the acidic milieu of phagolysosomes. Our studies on podosome formation of cells adhered to fibrinogen revealed that CR3 and to some extent CR4 localize in the close vicinity of podosome cores, however, we found a different podosome arrangement in the case of MDDCs compared to monocytes and MDMs.

\section{Materials and Methods}

\subsection{Isolation of human monocytes and neutrophil granulocytes.}

Monocytes were isolated from buffy coat obtained from healthy donors and provided by the Hungarian National Blood Transfusion Service. Informed consent was provided for the use of blood samples according to the Helsinki Declaration. Peripheral blood mononuclear cells (PBMC) were separated by Ficoll-Paque PLUS (GE Healthcare Life Sciences) density gradient centrifugation and monocytes were isolated negatively by using the Miltenyi Monocyte Isolation Kit II. Purity of isolated monocytes was analysed by flow cytometry using anti-CD14 antibody. 
For the isolation of neutrophil granulocytes PBMC were removed by Ficoll-Paque PLUS density gradient centrifugation, then dextran sedimentation was performed using Dextran T500 (Pharmacia Fine Chemicals, Uppsala, Sweden). Red blood cells were lysed in hypotonic buffer. Purity of isolated neutrophils was analysed by flow cytometry using anti-CD16 and anti-CD14 antibodies.

\subsection{Generation of monocyte-derived macrophages (MDMs) and monocyte-derived dendritic cells (MDDCs)}

To generate MDDCs and MDMs, monocytes were isolated with Miltenyi CD14 MicroBeads from PBMC. Cells were cultivated in RPMI-1640 Medium (Sigma-Aldrich) supplemented with gentamicin (Sigma-Aldrich) and 10 \% FCS (Sigma-Aldrich). To generate MDMs 100 $\mathrm{ng} / \mathrm{mL} \mathrm{rHu}$ GM-CSF (R\&D systems) was added to the isolated monocytes. To generate MDDCs $100 \mathrm{ng} / \mathrm{mL} \mathrm{rHu}$ GM-CSF (R\&D systems) and $15 \mathrm{ng} / \mathrm{mL} \mathrm{rHu}$ IL-4 (R\&D systems) were added to the monocytes. Cytokines were resupplied on day 3 of differentiation. Studies were carried out on day 5 .

\subsection{Phagocytosis of Staphylococcus aureus}

To test the phagocytic capacity of immune cells Staphylococcus aureus (Wood strain without protein A) BioParticles (Molecular Probes) conjugated with Alexa488 or pHrodo Green was used. Staphylococcus was opsonized with normal human serum for $1 \mathrm{~h}$ at $37^{\circ} \mathrm{C}$, then washed extensively and offered to the cells under different conditions. During opsonization, S. aureus activates the alternative pathway of the complement system, and $\mathrm{iC} 3 \mathrm{~b}$, the main ligand of CR3 and CR4, is deposited on its surface (Turner, Grant, Seymour, Harvey, \& Levinsky, 1986; Sándor 2013). In blocking experiments the anti-CD11b (monoclonal mouse IgG1 clone TMG6-5, provided by István Andó at BRC Szeged, Hungary) or anti-CD11c antibody (monoclonal mouse IgG1 clone 3.9, Biolegend) was used. Both antibodies are specific for the ligand binding domain of the corresponding integrin and were used in sterile, azide-free form at saturating concentration previously titrated by flow cytometry. The effect of receptor specific antibodies was compared to samples incubated with an isotype matched control antibody (mouse IgG1 clone MOPC-21, Biolegend). To exclude Fc-receptor mediated phagocytosis and the binding of the blocking monoclonal antibodies we used an Fc-receptor blocking reagent (Miltenyi Biotech). All reagents were diluted in Hank's Balanced Salt Solution (HBSS) buffer supplemented with 5\% FCS and 5 $\mathrm{mM}$ glutamine, $\mathrm{pH}$ 7.4. The cells were incubated with the blocking antibodies for $30 \mathrm{~min}$ on ice before the phagocytosis. Without a washing step S. aureus was offered to them at 200 $\mu \mathrm{g} / \mathrm{ml}$ concentration for $90 \mathrm{~min}$ at $37^{\circ} \mathrm{C}$ in a $\mathrm{CO} 2$ incubator or on ice. After $90 \mathrm{~min}$, phagocytosis was stopped by washing the samples with ice cold PBS and placing the cells on ice. Samples were analysed immediately by flow cytometry using BD FACS Calibur flow cytometer and CellQuest software for data acquisition and FCS Express 3.0 software for data analysis. 


\subsection{Analysis of the contact surface by confocal microscopy}

Generation of the adhesive surface and adherent cell layer. The wells of a CELLview cell culture dish with glass bottom (Greiner) were coated with $10 \mu \mathrm{g} / \mathrm{ml}$ fibrinogen (Merck) in phosphate buffered saline (PBS) solution for 1 hour at $37{ }^{\circ} \mathrm{C}$, free surfaces were blocked with synthetic copolymer poly(L-lysine)-graft-poly(ethylene glycol) (PLL-g-PEG, SuSoS AG) for $30 \mathrm{~min}$ at $37^{\circ} \mathrm{C}$. After washing, $10^{4}$ cells in RPMI1640 supplemented with $10 \%$ FCS were plated onto the adhesive surface. Cells were let to adhere for $30 \mathrm{~min}$ at $37^{\circ} \mathrm{C}$ in a $\mathrm{CO}_{2}$ incubator, then fixed with $2 \%$ paraformaldehyde for $10 \mathrm{~min}$ at $37^{\circ} \mathrm{C}$. Unbound cells were removed by washing two times with PBS.

Staining of the actin cytoskeleton. To analyse the podosome formation on the contact zone the actin cytoskeleton of adhered cells was stained with phalloidin-Alexa488 (Molecular Probes, Invitrogen) diluted 1:100 in PBS-0,1\% Triton X-100 (Reanal) for 15 minutes, then washed 2 times with PBS. Nuclei were stained with Draq5 (BioLegend) diluted 1:1000 in PBS for $15 \mathrm{~min}$. Samples were analysed by an Olympus IX81 confocal microscope (60x objective) applying Fluowiev500 confocal workstation.

Staining of CD11b/CD11c on adherent cells. Fixed samples were permeabilized with $0.1 \%$ Tween-20 (Reanal) in PBS for $20 \mathrm{~min}$ at $37^{\circ} \mathrm{C}$. The permeabilizing solution was replaced with a blocking solution: $0.2 \%$ gelatine (Merck) in $0.1 \%$ Tween-20 in PBS and incubated for 1 hour at $37^{\circ} \mathrm{C}$. The antibodies were diluted in the blocking solution; primary antibodies were incubated overnight at $37^{\circ} \mathrm{C}$, secondary antibodies for 2 hours at $37^{\circ} \mathrm{C}$. Cells were washed with $0.1 \%$ Tween-20 in PBS 3 times for 5 minutes before adding the secondary antibody. Antibodies used for staining: anti-CD11b (EP1345Y, Abcam), anti-CD11c (EP1347Y, Abcam), goat anti-rabbit IgG-A647 (Molecular Probes). After the secondary antibody staining cells were washed 3 times for 5 minutes with $0.1 \%$ Tween-20 in PBS. The actin cytoskeleton was stained with phalloidin-Alexa488 (Molecular Probes) 1:80 diluted in 0.1\% Tween-20 PBS for 45 minutes at $37^{\circ} \mathrm{C}$. Samples were analysed by an Olympus IX81 confocal microscope (60x objective) applying Fluowiev500 confocal workstation.

\subsection{Statistics}

One way ANOVA with Tukey's post-test and unpaired t-test was performed with GraphPad software, $\mathrm{p}<0.05$ was considered significant. 


\section{Results}

\subsection{The role of CR3 and CR4 in the phagocytosis of various human phagocytes}

The phagocytic capacity of monocytes, MDDCs, DCs and granulocytes was measured using Staphylococcus aureus conjugated to Alexa488 or pHrodo Green dye. Using bacteria labelled with Alexa488 both surface bound and internalized particles can be detected, while employing the $\mathrm{pH}$ sensitive $\mathrm{pHrodo}$ Green dye, bacteria are only visible when they reach the acidic milieu of phagolysosomes on the degradative pathway. Comparing the activity of MDMs and DCs we found a significant difference in the amount of phagocytosed bacteria measured by Alexa488 (Fig.1C) and pHrodo Green (Fig.1D) conjugated S. aureus. The lower phagocytic capacity of MDDCs is in agreement with their major role in antigen presentation (Savina 2007). In the control samples, where cells were kept on ice, a lower level of binding could be observed (Fig.1A) which was not prominent for the pHrodo Green labelled bacteria (Fig.1B).

To determine the participation of CR3 and CR4 in the uptake of opsonized particles, cells were treated with ligand binding site specific blocking antibodies before and during incubation with the bacteria. The phagocytosis assay was carried out using iC $3 b$ opsonized $S$. aureus. In the presence of the CD11b specific antibody monocytes and MDMs showed decreased phagocytosis when employing Alexa488 or PHrodo Green labelled bacteria. (Fig.2). However, when macrophages were treated with the CD11c specific antibody, a significant reduction in the binding of Alexa488 conjugated S. aureus (Fig.2C) could be observed. This blocking effect however was not present when pHrodo Green S. aureus was used (Fig.2D). These findings demonstrate that CD11c participates in the binding of iC3b opsonized S. aureus, while further steps leading to the digestion of the coccus are mediated by CD11b in macrophages.

In accordance with our previous results (Sándor, Immunobiology, 2013), blocking of CR3 on MDDCs inhibited the phagocytosis of both Alexa488 (Fig.3A) and pHrodo Green (Fig.3B) conjugated bacteria, whereas inhibiting CD11c had no effect. Experiments with neutrophil granulocytes (Fig.3C-D) showed similar results, namely, blocking of CD11b caused a decrease in phagocytosis. 
A

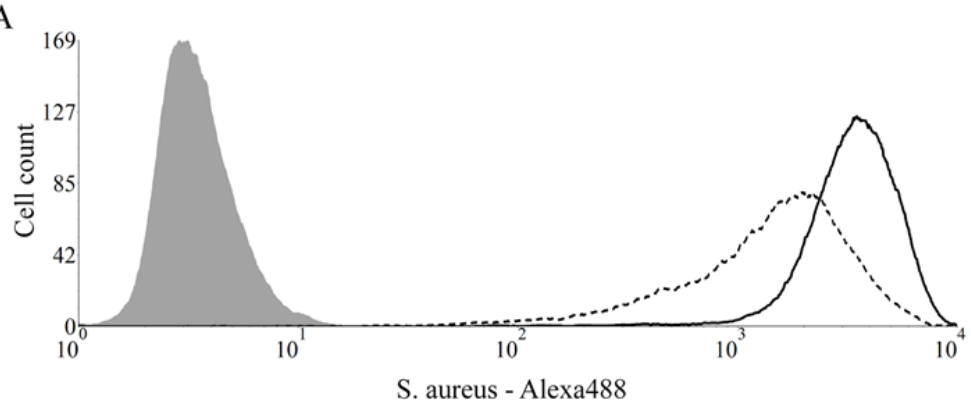

B

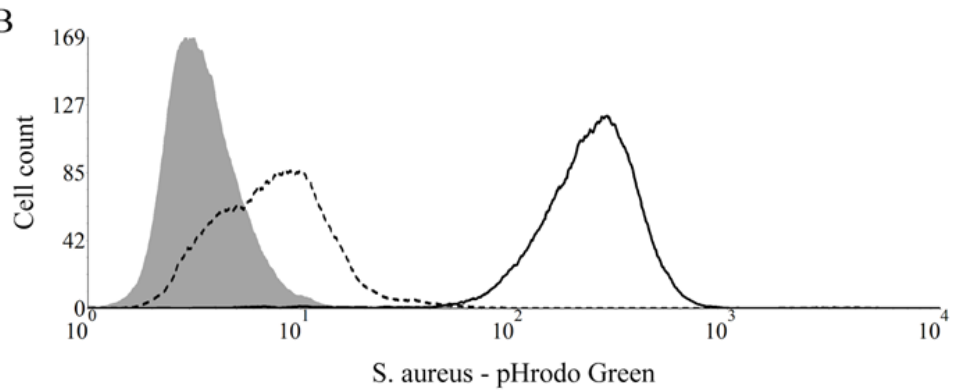

$\mathrm{C}$

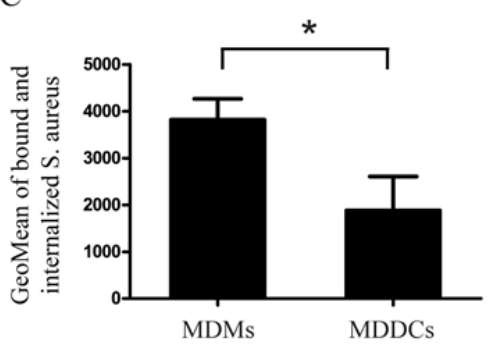

$\mathrm{D}$

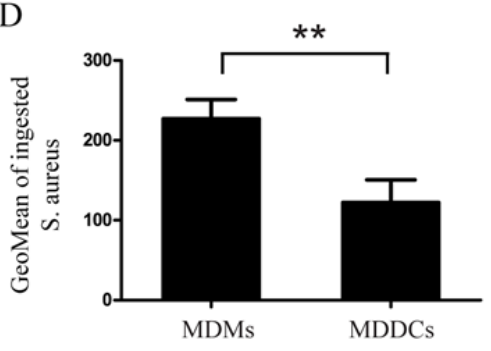

Figure 1. Fluorescence intensity of bound/internalized and digested bacteria by MDMs and MDDCs. Panel $\mathrm{A}$ and $\mathrm{C}$ : results obtained employing $S$. aureus conjugated with Alexa488 ; panel B and D: results obtained with S. aureus conjugated with pHrodo Green dye. Representative histograms of 5 (A) and 5 (B) independent experiments performed on MDMs are shown. Histograms: grey background: autofluorescence; dashed line: negative control (cells kept on ice); black line: isotype control (cells were kept at 37 in $\mathrm{CO}_{2}$ incubator during phagocytosis). Graphs $\mathrm{C}$ and $\mathrm{D}$ show the geometric mean fluorescence intensity of bound and digested S. aureus by MDMs and MDDCs, respectively. Mean+/- SD of 3 independent experiments are shown. Unpaired t-test was used to determine significant differences, $*=\mathrm{p}<0.05 ; * *=\mathrm{p}<0.01$. 


\section{Monocytes}

A

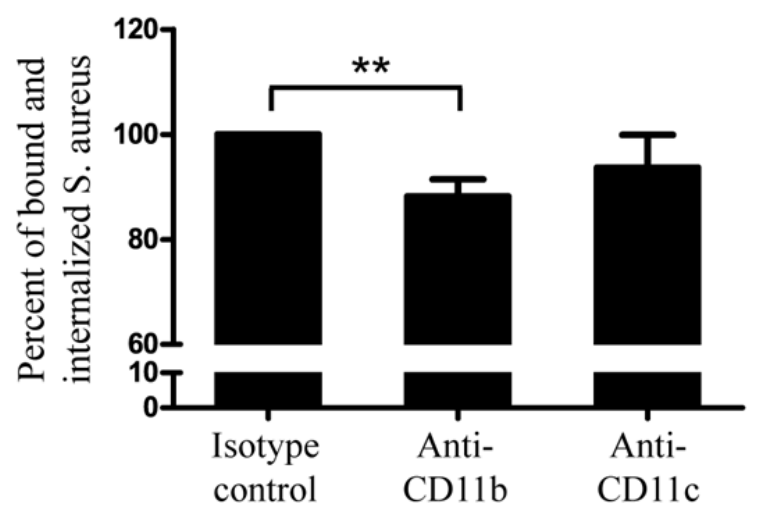

B

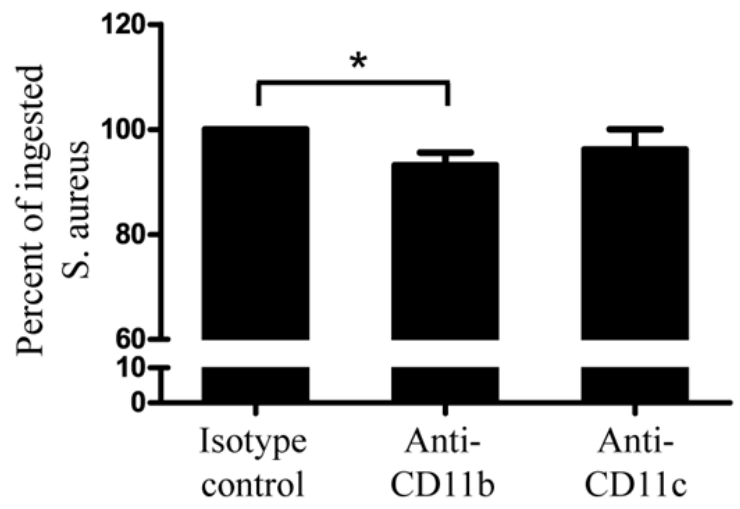

\section{MDMs}

C

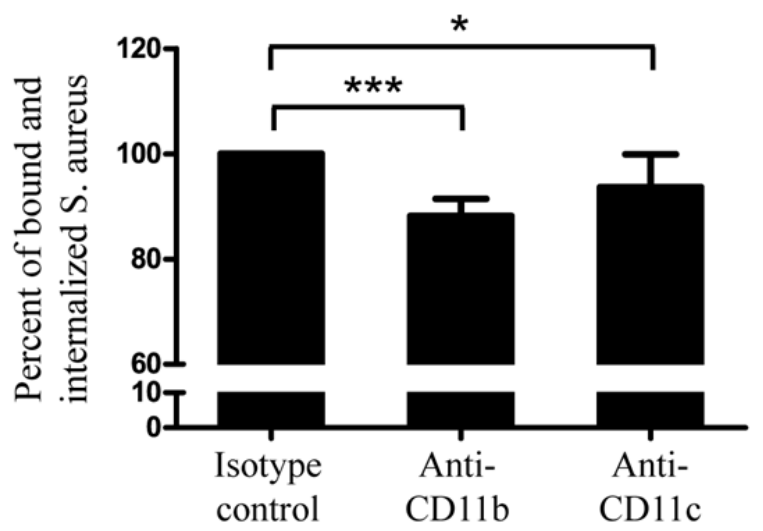

D

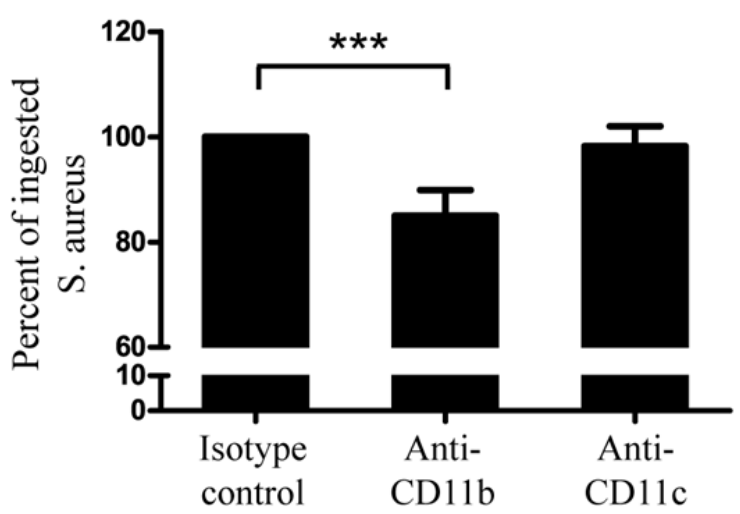

Figure 2. Phagocytic capacity of monocytes and MDMs after blocking CD11b or CD11c with ligand-binding site specific antibodies. Using bacteria labelled with Alexa488 (A and C) both surface bound and internalized particles are detected. With the $\mathrm{pH}$ sensitive pHrodo Green dye (B and D) bacteria are only visible when they reach the acidic phagolysosomes. The amount of bound/internalized, vs. digested fluorescently labelled bacteria was measured by flow cytometry. The effect of antibody treatment was compared to isotype control samples, shown as $100 \%$. For monocytes (A and B) results of 4 donors are shown, mean $+/-$ SD, for MDMs (C and D) 6 (C) and 4 (D) donors' results (mean+/- SD) are shown. One way ANOVA with Tukey's post-test was used to determine significant differences compared to control, $*=\mathrm{p}<0.05 ; * *=\mathrm{p}<0.01 ; * * *=\mathrm{p}<0.001$. 


\section{MDDCs}

A

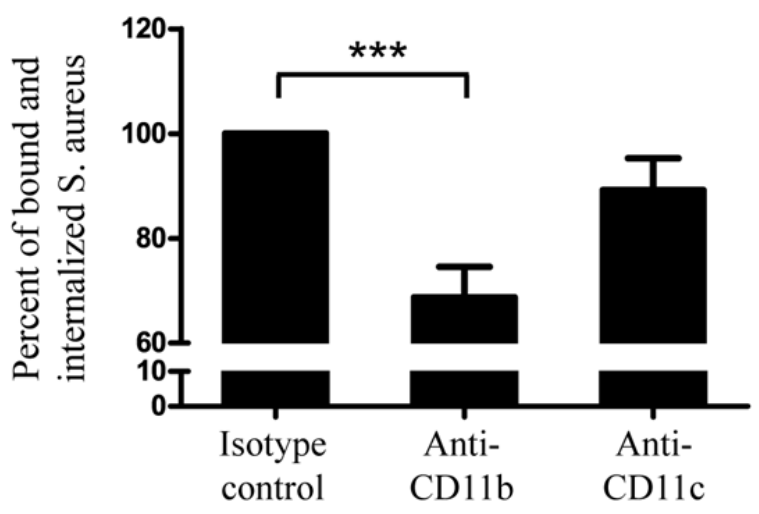

B

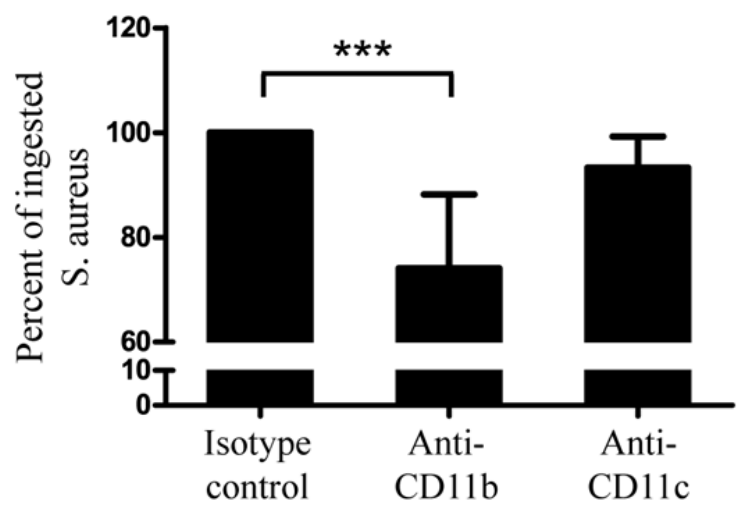

\section{Neutrophil granulocytes}

C

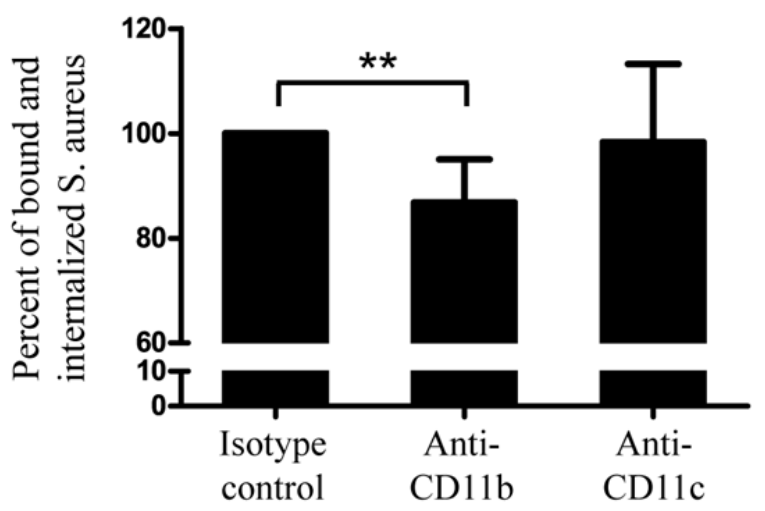

D

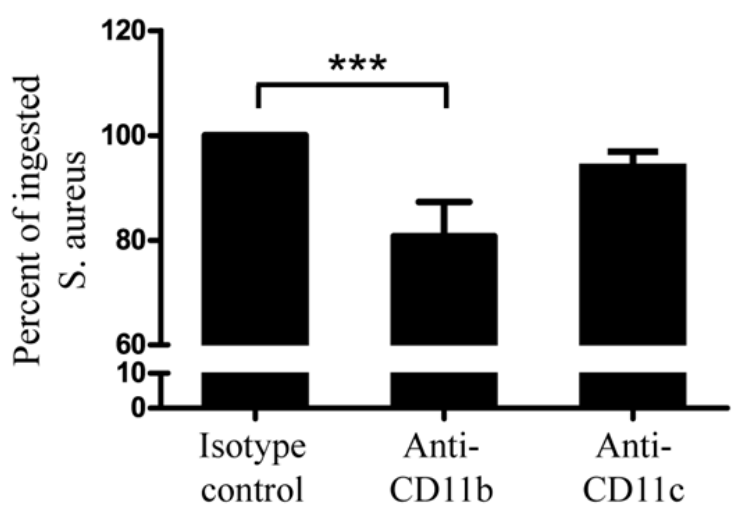

Figure 3. Phagocytic capacity of MDDCs and neutrophil granulocytes after blocking CD11b or CD11c with ligand-binding site specific antibodies. Using bacteria labelled with Alexa488 (A and C) both surface bound and internalized particles are detected. With the $\mathrm{pH}$ sensitive pHrodo Green dye (B and D) bacteria are only visible when they reach the acidic phagolysosomes. The amount of bound/digested fluorescently labelled bacteria was measured by flow cytometry. The effect of antibody treatment was compared to isotype control samples, shown as 100\%. For MDDCs (A and B) results of 3 (A) and 6 (B) independent donors (mean+/- SD) are shown, for neutrophil granulocytes (C and D) 14 (C) and 4 (D) independent donors' results (mean+/- SD) are shown. One way ANOVA with Tukey's posttest was used to determine significant differences compared to control, $* *=p<0.01$; $* * *=\mathrm{p}<0.001$ 


\subsection{Adhesive structures of human phagocytes - podosome patterns}

Podosomes can be revealed by staining the actin cytoskeleton with phalloidin-Alexa488 (Fig.4). The actin core of these structures appears as a small, strongly fluorescent dot on confocal microscopy images. We found characteristic podosome patterns in the case of various human phagocytes. Smaller monocytes (Fig.4A) displayed an even distribution across the substrate contacting surface. The podosomes of macrophages (Fig.4B) showed either a similar distribution, or a belt like pattern. In dendritic cells however (Fig.4C) podosomes are arranged in clusters or accumulate in the leading edge of polarized cells. Neutrophil granulocytes (Fig.4D) also have an even distribution of actin cores.
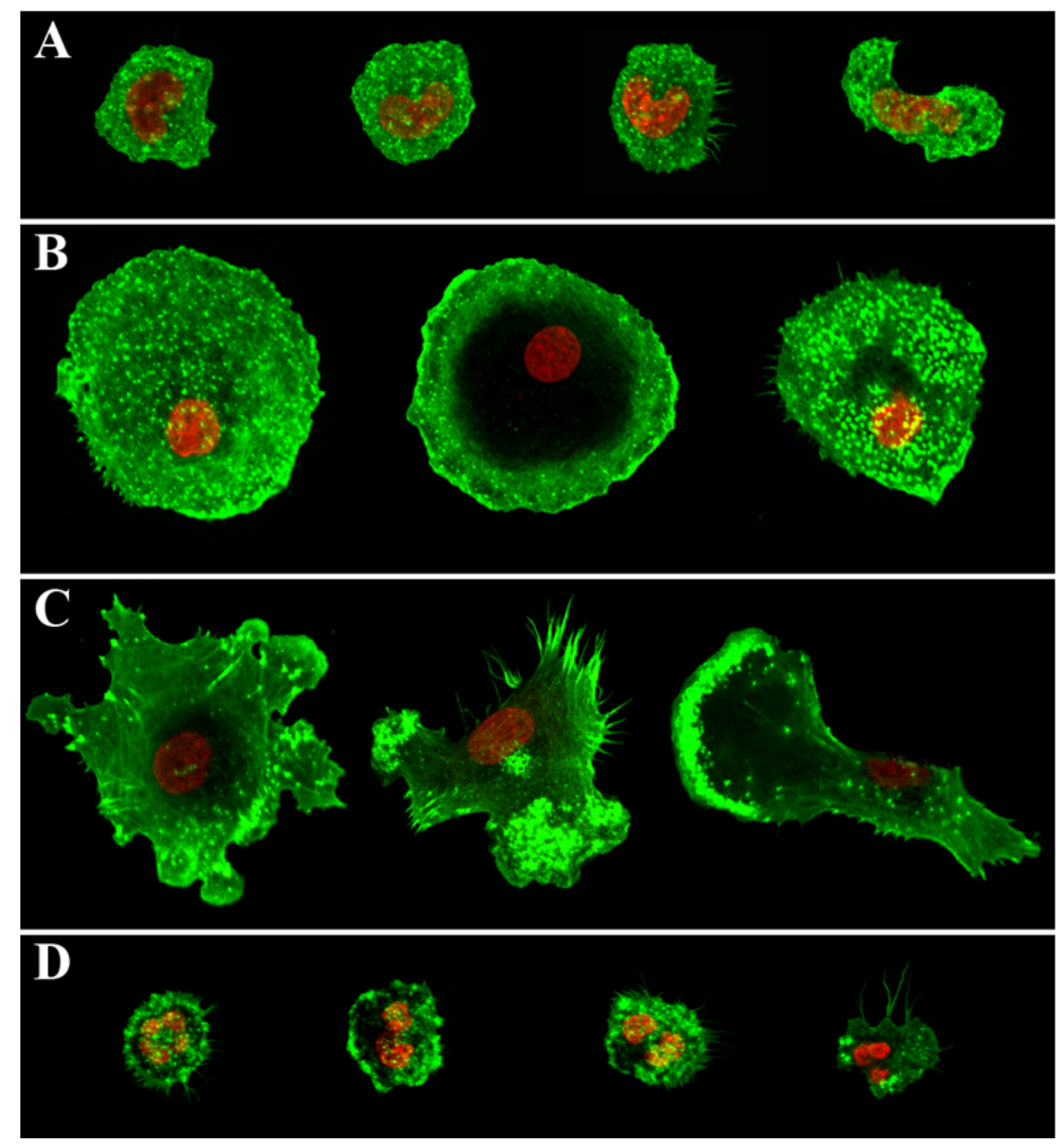

Figure 4. Podosome patterns of human phagocytes attached to fibrinogen. Different podosome formation can be observed in the case of human monocytes (A), MDMs (B), MDDCs (C) and neutrophil granulocytes (D).Cells were let to adhere for 30 minutes on a fibrinogen coated surface, fixed with paraformaldehyde and unbound cells were washed away. The actin cytoskeleton was stained with phalloidin- Alexa488 (shown in green), nuclei were stained with Draq5 (shown in red). Representative images were chosen from at least 3 independent experiments. 


\subsection{Localization of CD11b and CD11c on the contact surface of adherent cells}

Integrins are known to participate in cell adhesion to various substrates, including extracellular matrix components. In our experiments we employed fibrinogen, a ligand commonly used to study the functions of CR3 and CR4. Since fibrinogen coated surfaces are known to induce the formation of podosomes, myeloid cells were let to adhere as described in the section of Materials and methods, and the actin core was visualized using phalloidinAlexa488 staining (Fig.5A,D,G,J). The immunocytochemical staining of CD11b and CD11c showed a wider localization than actin, which is in correspondence with data showing an adhesion ring around the core of podosomes (Schachtner, cytoskeleton, 2013;Calle, European journal of cell biology, 2006). Confocal microscopy analysis revealed the presence of both CD11b (Fig.5C,I) and CD11c (Fig.5F,L) in the podosome adhesion ring on the contact surface of MDMs and MDDCs.
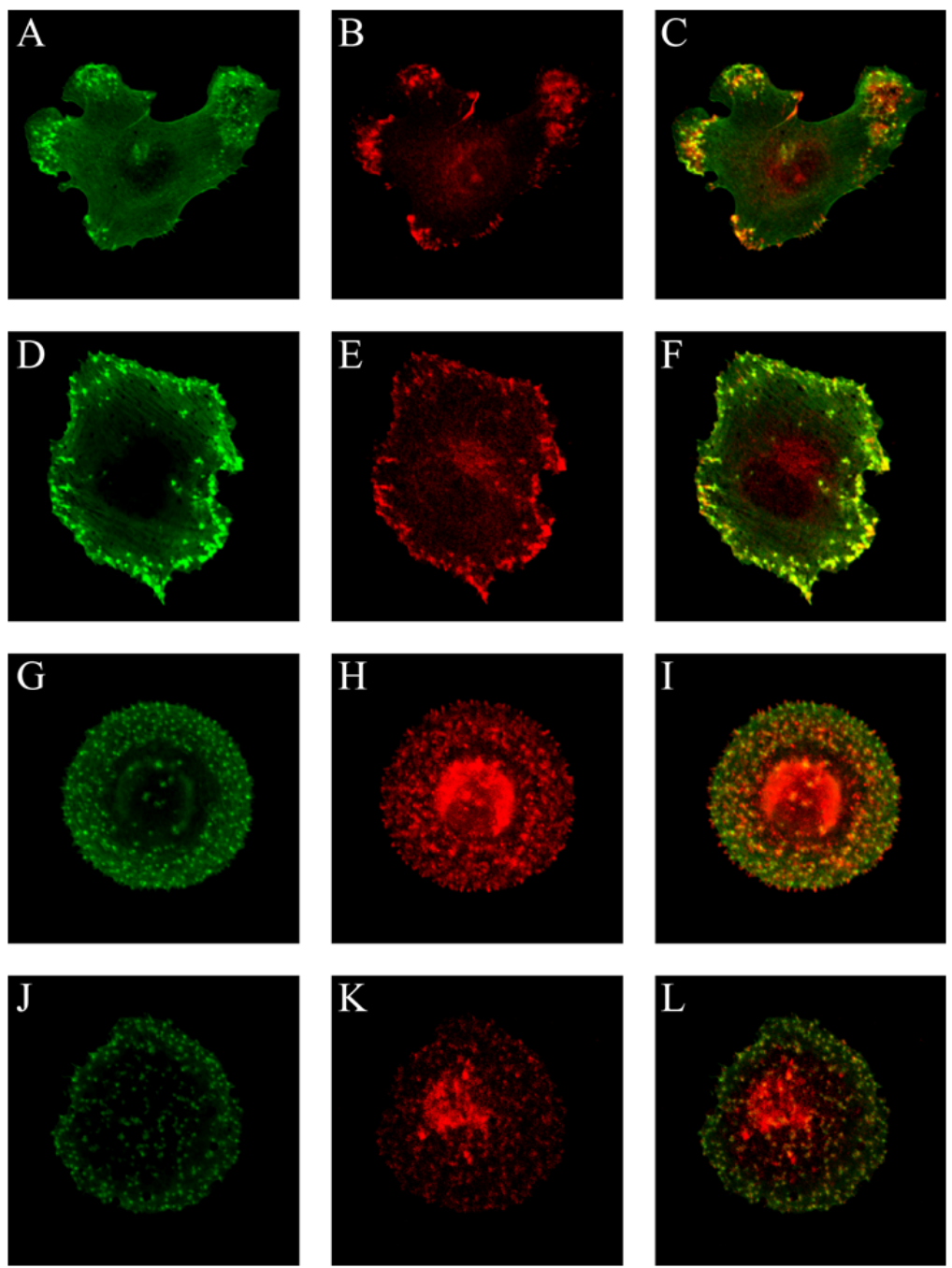

Figure 1. Localization of CD11b and CD11c on the contact surface of human myeloid cells. MDDCs (A-F) and MDMs (G-L) were plated to a fibrinogen coated surface, followed by staining with phalloidin-Alexa488 (A,D,G,J), anti-CD11b $(\mathrm{B}, \mathrm{H})$ or anti-CD11c $(\mathrm{E}, \mathrm{K})$. Merged images are shown in C,F,I,L. CD11b and CD11c are located around the actin core of podosomes in both cell types. Images of one representative experiment out of 3 are shown. 


\section{Discussion}

CR3 and CR4 are generally thought to mediate similar functions due to their structural homology and overlapping ligand specificity. The extracellular regions of $\mathrm{CD} 11 \mathrm{~b}$ and CD11c, display 87\% similarity, but their intracellular domains, important for signal transduction and connection with the cytoskeleton, differ in length and amino acid sequence displaying only 56\% identity (Corbi et al. 1988; Ross et al. 1992). The two receptors have different distribution and expression pattern in mouse and human cells, consequently data obtained in mouse systems can be applied for human immune cells only with great caution (Miller, Schwarting, and Springer 1986). In mice CR4 is expressed on DCs in the highest number, and it is often used as a marker for these cells. Very recent data suggests however, that under inflammatory conditions mouse monocytes and macrophages also express CR4, which exerts antimicrobial functions (Jawhara, Pluskota, Cao, Plow, \& Soloviev, 2017). In humans both receptors are present on monocytes, macrophages, DCs, neutrophils and NK cells.(Mazzone 1995, Haematologica) The simultaneous presence of these receptors on leukocytes and their different signalling properties however suggest a separation of functions. Here we discuss the functional differences we found between CR3 and CR4 in the uptake and digestion of $\mathrm{iC} 3 \mathrm{~b}$ opsonized $S$. aureus and demonstrate the role of these receptors in podosome formation by human MDMs, MDDCs, monocytes and neutrophils.

The opsonophagocytic function of CR3 in macrophages has been proven earlier (Aderem, 1999), and the major role of CR4 in the uptake of Mycobacterium tuberculosis by alveolar macrophages has also been demonstrated (Hirsch, Ellner, Russell, \& Rich, 1994). Though alveolar macrophages differ from monocyte derived macrophages due to their tissue microenvironment and cytokine milieu, several data show that on MDMs both CR3 and CR4 participate in the phagocytosis of opsonized Cryptococcus neoformans ((Levitz, 2002), Mycobacterium leprae (L S Schlesinger \& Horwitz, 1991) and Francisella tularensis (Schwartz et al., 2012). These studies however do not clearly distinguish between the complement receptor mediated binding and digestion of the pathogens. Now we compared these two different functions using the $\mathrm{pH}$ sensitive $\mathrm{pHrodo}$ Green labelled Staphylococcus aureus and the classical Alexa488 conjugated particles. This approach allowed us to detect bacteria destined for degradation and separate those from the endocytosed ones. Employing this method, in the case of MDMs we found a collaboration between the two complement receptors. Namely, we observed that blocking CR4 decreased the amount of surface bound particles, while the digestion of iC3b opsonized S. aureus was dependent on functional CR3.

Our group demonstrated earlier that blocking of CD11c did not influence the uptake of opsonized yeast or S. aureus by MDDCs. We confirmed these results using siRNA; when downregulation of CD11b blocked the phagocytosis of opsonized particles while silencing CD11c had no effect on their uptake (Sándor et al., 2013). Now we have strengthened these findings by showing that only CR3 blocking decreases the binding and uptake of $S$. aureus. In contrast to this Ben Nasr et al. demonstrated that blocking both CR3 or CR4 inhibited the phagocytosis of opsonized, live Francisella tularensis by human MDDCs.(Ben Nasr et al., 
2006). This contradiction might be caused by differences in the pathogens and the capacity of live Francisella tularensis to utilize CR4 as well to get into the host cells.

Studies of phagocytosis carried out employing monocytes and neutrophils underline the importance of CR3 expressed by these cells. Monocytes utilize CR3 but not CR4, for the phagocytosis of Mycobacterium tuberculosis (Hirsch, Ellner, Russell, \& Rich, 1994) and M. leprae (Schlesinger \& Horwitz, 1990), similarly to neutrophils in the case of Francisella tularensis (Schwartz et al., 2012). In our present study we show that in the phagocytosis of the extracellular $S$. aureus opsonized by iC3b, CR3 plays a dominant role over CR4 by both monocytes and neutrophils.

In addition to being a phagocytic receptor, CR3 and CR4 are known to mediate cell adhesion, spreading and migration through the establishment of cell-cell and cellextracellular matrix connections (Hynes, Cell, 2002). Podosomes are known to mediate shortlived adhesion spots that are formed and quickly remodelled during migration. These structures have an F-actin core surrounded by an adhesion ring (Linder \& Aepfelbacher 2003) and can be arranged in clusters, rings or belts (Linder \& Wiesner 2015). Podosomes are characteristic for monocytic cells, such as monocytes, macrophages, osteoclasts and dendritic cells. Here we studied the podosomes of human phagocytes adhered to fibrinogen coated surface. Monocytes and MDMs mostly showed an even distribution of podosomes scattered across the contact surface, however a belt like pattern could also be observed for MDMs. The podosomes of dendritic cells were found to be arranged in clusters, or condensed in the leading edge of migrating cells. Whether human neutrophils have podosomes is still debated. Neutrophils isolated from mice and pretreated with fMLP formed podosome-like structures, containing and actin core and a vinculin ring, on fibrinogen coated slips (Szczur, 2006). In this study we detected the formation of actin rich dots evenly spread on the contact surface of human neutrophils adhered to fibrinogen.

The differences in podosome formation by various cell types could be explained by the different milieu and motility of the investigated cells. Monocytes and neutrophils circulate in the bloodstream and adhere to vessel walls when they reach the site of infection. They migrate through the endothelium into the injured tissue, whereas DCs move from tissues to lymph nodes through lymphatic vessels after antigen uptake (Calle 2008). During this journey DCs go through maturation and lose their capacity for phagocytosis (Savina 2007) as well as podosome formation, because of changes in cytoskeletal reorganization (Burns 2004). Thus, podosome formation might be restricted to cells with an actively migrating phenotype.

The importance of $\beta_{2}$ integrins in podosome formation and podosome mediated adhesion has been proven, but the individual role of these receptors had not been studied so far. Burns et al. found, that $\beta_{2}$ integrins are specifically recruited to podosomes in human MDDCs on a fibronectin surface (a ligand for both $\beta_{1}$ and $\beta_{2}$ integrins), whereas $\beta_{1}$ integrins show a disperse distribution (Burns, Cell Motility and the Cytoskeleton, 2004). In a $\beta_{2}$ integrin-null mouse model, Gawden-Bone et al. showed that in the absence of $\beta_{2}$ integrins podosome assembly is disrupted. (Gawden-Bone, Journal of Cell Science, 2014).

Here we show that both CR3 and CR4 are located in the adhesion ring of podosomes in human MDMs and MDDCs attached to fibrinogen. Additionally, a strong staining for the receptors can be observed inside the cells, around the nucleus. Integrins are known to rapidly recycle between the cell membrane and endosomes (Bretscher 1992), which can occur via 
distinct routes (Arjonen et al. 2012; Jones et al. 2006). In the long loop of recycling integrins go through the perinuclear recycling compartment before returning to the cell surface (Caswell et al. 2009). No data is available yet on the exact recycling route used by $\beta 2$ integrins, but the staining pattern we found around the nucleus might implicate a passage through the perinuclear recycling compartment.

Taking together, we obtained further evidence supporting our view that CR3 and CR4 exert distinct functions in human phagocytes. Though both CR3 and CR4 are able to bind fibrinogen and $\mathrm{iC} 3 \mathrm{~b}$, we found that either of them dominates the other in various functions, such as antigen uptake, digestion, in addition to adhesion and spreading, which we had shown earlier (Sándor, 2016).

\section{Acknowledgements}

The CD11b ligand bindig site specific monoclonal antibody TMG6-5 (mIgG1) was kindly provided by István Andó, BRC, Szeged, Hungary.

Financial support from Hungarian Science Fund OTKA grant no. 104838 and 112011 is gratefully acknowledged.

\section{References}

[1] H. Rosen, S.K. Law, The leukocyte cell surface receptor(s) for the iC3b product of complement., Curr. Top. Microbiol. Immunol. 153 (1990) 99-122. http://www.ncbi.nlm.nih.gov/pubmed/2137074.

[2] G.D. Ross, V. Větvicka, CR3 (CD11b, CD18): a phagocyte and NK cell membrane receptor with multiple ligand specificities and functions., Clin. Exp. Immunol. 92 (1993) 181-4. doi:10.1111/j.1365-2249.1993.tb03377.x.

[3] V.K. Lishko, V.P. Yakubenko, K.M. Hertzberg, G. Grieninger, T.P. Ugarova, The alternatively spliced alpha(E)C domain of human fibrinogen-420 is a novel ligand for leukocyte integrins alpha(M)beta(2) and alpha(X)beta(2)., Blood. 98 (2001) 244855. doi:10.1182/blood.V98.8.2448.

[4] M.S. Diamond, J. Garcia-Aguilar, J.K. Bickford, A.L. Corbi, T.A. Springer, The I domain is a major recognition site on the leukocyte integrin Mac-1 (CD11b/CD18) for four distinct adhesion ligands., J. Cell Biol. 120 (1993) 1031-43. doi:10.1083/jcb.120.4.1031.

[5] M.J. Flick, X. Du, D.P. Witte, M. Jirousková, D. a Soloviev, S.J. Busuttil, E.F. Plow, J.L. Degen, Leukocyte engagement of fibrin(ogen) via the integrin receptor alphaMbeta2/Mac-1 is critical for host inflammatory response in vivo., J. Clin. Invest. 113 (2004) 1596-606. doi:10.1172/JCI20741. 
[6] R.I. Thacker, G.S. Retzinger, Adsorbed fibrinogen regulates the behavior of human dendritic cells in a CD18-dependent manner., Exp. Mol. Pathol. 84 (2008) 12230. doi:10.1016/j.yexmp.2008.01.001.

[7] N. Sándor, S. Lukácsi, R. Ungai-Salánki, N. Orgován, B. Szabó, R. Horváth, A. Erdei, Z. Bajtay, CD11c/CD18 Dominates Adhesion of Human Monocytes, Macrophages and Dendritic Cells over CD11b/CD18., PLoS One. 11 (2016) e0163120. doi:10.1371/journal.pone.0163120.

[8] S. Burns, A.J. Thrasher, M.P. Blundell, L. Machesky, G.E. Jones, Configuration of human dendritic cell cytoskeleton by Rho GTPases, the WAS protein, and differentiation., Blood. 98 (2001) 1142-9. doi:10.1182/blood.V98.4.1142.

[9] Y. Calle, S. Burns, A.J. Thrasher, G.E. Jones, The leukocyte podosome., Eur. J. Cell Biol. 85 (2006) 151-7. doi:10.1016/j.ejcb.2005.09.003.

[10] S. Linder, C. Wiesner, Tools of the trade: podosomes as multipurpose organelles of monocytic cells., Cell. Mol. Life Sci. 72 (2015) 121-35. doi:10.1007/s00018-014-1731-z.

[11] A.G. Dupuy, E. Caron, Integrin-dependent phagocytosis: spreading from microadhesion to new concepts., J. Cell Sci. 121 (2008) 1773-83. doi:10.1242/jcs.018036.

[12] L.M. Stuart, R.A.B. Ezekowitz, Phagocytosis: elegant complexity., Immunity. 22 (2005) 539-50. doi:10.1016/j.immuni.2005.05.002.

[13] Z. Bajtay, C. Speth, A. Erdei, M.P. Dierich, Cutting edge: productive HIV-1 infection of dendritic cells via complement receptor type 3 (CR3, CD11b/CD18)., J. Immunol. 173 (2004) 4775-8. doi:10.4049/jimmunol.173.8.4775.

[14] C.R. Oliva, M.K. Swiecki, C.E. Griguer, M.W. Lisanby, D.C. Bullard, C.L. Turnbough, J.F. Kearney, The integrin Mac-1 (CR3) mediates internalization and directs Bacillus anthracis spores into professional phagocytes., Proc. Natl. Acad. Sci. U. S. A. 105 (2008) 1261-6. doi:10.1073/pnas.0709321105.

[15] M. Nagl, L. Kacani, B. Müllauer, E.-M. Lemberger, H. Stoiber, G.M. Sprinzl, H. Schennach, M.P. Dierich, Phagocytosis and killing of bacteria by professional phagocytes and dendritic cells., Clin. Diagn. Lab. Immunol. 9 (2002) 1165-8. doi:10.1128/CDLI.9.6.1165-1168.2002.

[16] A. Savina, S. Amigorena, Phagocytosis and antigen presentation in dendritic cells, Immunol. Rev. 219 (2007) 143-156. doi:10.1111/j.1600-065X.2007.00552.x.

[17] N. Sándor, K. Kristóf, K. Paréj, D. Pap, A. Erdei, Z. Bajtay, CR3 is the dominant phagocytotic complement receptor on human dendritic cells., Immunobiology. 218 (2013) 652-63. doi:10.1016/j.imbio.2012.07.031.

[18] M.W. Turner, C. Grant, N.D. Seymour, B. Harvey, R.J. Levinsky, Evaluation of $\mathrm{C} 3 \mathrm{~b} / \mathrm{C} 3 \mathrm{bi}$ opsonization and chemiluminescence with selected yeasts and bacteria using sera of different opsonic potential, Immunology. 58 (1986) 111-115. http://www.ncbi.nlm.nih.gov/entrez/query.fcgi?cmd=Retrieve \&db=PubMed\&dopt=Cit ation\&list_uids $=3519441$.

[19] H. Schachtner, S.D.J. Calaminus, S.G. Thomas, L.M. Machesky, Podosomes in adhesion, migration, mechanosensing and matrix remodeling., Cytoskeleton (Hoboken). 70 (2013) 572-89. doi:10.1002/cm.21119. 
[20] A.L. Corbi, T.K. Kishimoto, L.J. Miller, T.A. Springer, The human leukocyte adhesion glycoprotein Mac-1 (complement receptor type 3, CD11b) alpha subunit. Cloning, primary structure, and relation to the integrins, von Willebrand factor and factor B., J. Biol. Chem. $263 \quad$ (1988) 12403-11. http://www.ncbi.nlm.nih.gov/pubmed/2457584.

[21] G.D. Ross, W. Reed, J.G. Dalzell, S.E. Becker, N. Hogg, Macrophage cytoskeleton association with $\mathrm{CR} 3$ and CR4 regulates receptor mobility and phagocytosis of iC3b-opsonized erythrocytes., J. Leukoc. Biol. 51 (1992) 109-17. http://www.ncbi.nlm.nih.gov/pubmed/1358992.

[22] S. Jawhara, E. Pluskota, W. Cao, E.F. Plow, D.A. Soloviev, Distinct Effects of Integrins $\alpha \mathrm{X} \beta 2$ and $\alpha \mathrm{M} \beta 2$ on Leukocyte Subpopulations during Inflammation and Antimicrobial Responses., Infect. Immun. 85 (2017) 1-17. doi:10.1128/IAI.00644-16.

[23] L.J. Miller, R. Schwarting, T.A. Springer, Regulated expression of the Mac-1, LFA-1, p150,95 glycoprotein family during leukocyte differentiation., J. Immunol. 137 (1986) 2891-900. http://www.ncbi.nlm.nih.gov/pubmed/2428876.

[24] A. Aderem, D.M. Underhill, Mechanisms of phagocytosis in macrophages., Annu. Rev. Immunol. 17 (1999) 593-623. doi:10.1146/annurev.immunol.17.1.593.

[25] C.S. Hirsch, J.J. Ellner, D.G. Russell, E.A. Rich, Complement receptormediated uptake and tumor necrosis factor-alpha-mediated growth inhibition of Mycobacterium tuberculosis by human alveolar macrophages., J. Immunol. 152 (1994) 743-53. http://www.jimmunol.org/content/152/2/743.abstract.

[26] S.M. Levitz, Receptor-mediated recognition of Cryptococcus neoformans., Nihon Ishinkin Gakkai Zasshi. 43 (2002) 133-6. doi:10.3314/jjmm.43.133.

[27] L.S. Schlesinger, M. a Horwitz, Phagocytosis of Mycobacterium leprae by human monocyte-derived macrophages is mediated by complement receptors CR1 (CD35), CR3 (CD11b/CD18), and CR4 (CD11c/CD18) and IFN-gamma activation inhibits complement receptor function and phagocytosis of this bacte, J. Immunol. 147 (1991) 1983-94. http://www.ncbi.nlm.nih.gov/pubmed/1679838.

[28] J.T. Schwartz, J.H. Barker, M.E. Long, J. Kaufman, J. McCracken, L.-A.H. Allen, Natural IgM mediates complement-dependent uptake of Francisella tularensis by human neutrophils via complement receptors 1 and 3 in nonimmune serum., J. Immunol. 189 (2012) 3064-77. doi:10.4049/jimmunol.1200816.

[29] A. Ben Nasr, J. Haithcoat, J.E. Masterson, J.S. Gunn, T. Eaves-Pyles, G.R. Klimpel, Critical role for serum opsonins and complement receptors CR3 $(\mathrm{CD} 11 \mathrm{~b} / \mathrm{CD} 18)$ and CR4 (CD11c/CD18) in phagocytosis of Francisella tularensis by human dendritic cells (DC): uptake of Francisella leads to activation of immature DC and intracellular survival of, J. Leukoc. Biol. 80 (2006) 774-86. doi:10.1189/jlb.1205755.

[30] L.S. Schlesinger, M.A. Horwitz, Phagocytosis of leprosy bacilli is mediated by complement receptors $\mathrm{CR} 1$ and $\mathrm{CR} 3$ on human monocytes and complement component C3 in serum., J. Clin. Invest. 85 (1990) 1304-14. doi:10.1172/JCI114568.

[31] S. Linder, M. Aepfelbacher, Podosomes: adhesion hot-spots of invasive cells., Trends Cell Biol. 13 (2003) 376-85. doi:10.1016/S0962-8924(03)00128-4. 
[32] K. Szczur, H. Xu, S. Atkinson, Y. Zheng, M.-D. Filippi, Rho GTPase CDC42 regulates directionality and random movement via distinct MAPK pathways in neutrophils., Blood. 108 (2006) 4205-13. doi:10.1182/blood-2006-03-013789.

[33] C. Cougoule, E. Van Goethem, V. Le Cabec, F. Lafouresse, L. Dupré, V. Mehraj, J.-L. Mège, C. Lastrucci, I. Maridonneau-Parini, Blood leukocytes and macrophages of various phenotypes have distinct abilities to form podosomes and to migrate in 3D environments., Eur. J. Cell Biol. 91 (2012) 938-49. doi:10.1016/j.ejcb.2012.07.002.

[34] Y. Calle, I.M. Antón, A.J. Thrasher, G.E. Jones, WASP and WIP regulate podosomes in migrating leukocytes., J. Microsc. 231 (2008) 494-505. doi:10.1111/j.1365-2818.2008.02062.x.

[35] S. Burns, S.J. Hardy, J. Buddle, K.L. Yong, G.E. Jones, A.J. Thrasher, Maturation of DC is associated with changes in motile characteristics and adherence., Cell Motil. Cytoskeleton. 57 (2004) 118-32. doi:10.1002/cm.10163.

[36] C. Gawden-Bone, M. a West, V.L. Morrison, A.J. Edgar, S.J. McMillan, B.D. Dill, M. Trost, A. Prescott, S.C. Fagerholm, C. Watts, A crucial role for $\beta 2$ integrins in podosome formation, dynamics and Toll-like-receptor-signaled disassembly in dendritic cells., J. Cell Sci. 127 (2014) 4213-24. doi:10.1242/jcs.151167.

[37] M.S. Bretscher, Circulating integrins: alpha 5 beta 1, alpha 6 beta 4 and Mac1, but not alpha 3 beta 1, alpha 4 beta 1 or LFA-1., EMBO J. 11 (1992) 405-10. http://www.ncbi.nlm.nih.gov/pubmed/1531629.

[38] A. Arjonen, J. Alanko, S. Veltel, J. Ivaska, Distinct recycling of active and inactive $\beta 1$ integrins., Traffic. 13 (2012) 610-25. doi:10.1111/j.16000854.2012.01327.x.

[39] M.C. Jones, P.T. Caswell, J.C. Norman, Endocytic recycling pathways: emerging regulators of cell migration., Curr. Opin. Cell Biol. 18 (2006) 549-57. doi:10.1016/j.ceb.2006.08.003.

[40] P.T. Caswell, S. Vadrevu, J.C. Norman, Integrins: masters and slaves of endocytic transport., Nat. Rev. Mol. Cell Biol. 10 (2009) 843-53. doi:10.1038/nrm2799. 